\title{
Comparative Analysis of Domestic and Foreign Activation Methods of Technical Creativity
}

\author{
Valentina Bushueva \\ Bauman Moscow State Technical University \\ 5/1, 2nd Baumanskaya St. \\ Moscow, Russia 105005 \\ E-mail: vbush2008@rambler.ru
}

\author{
Nikolay Bushuev \\ Bauman Moscow State Technical University \\ 5/1, 2nd Baumanskaya St. \\ Moscow, Russia 105005 \\ E-mail: agrohim1@rambler.ru
}

\begin{abstract}
The purpose of the study was to substantiate the relevance of the comparative analysis of activation methods of technical creativity in domestic and foreign practice. The study focuses on the principles of development, advantages and disadvantages of both domestic and foreign activation methods, and underlines the importance of collective methods of activation in foreign practice. Within the research, the comparative analysis of domestic and foreign activation methods was done, where their application field was noted. This represents the scientific novelty of the study. Finally, some recommendations on the need to use domestic and foreign methods in view of the complexity of the problem being solved, its specifics, and the characteristics of the creative groups are given.
\end{abstract}

Keywords - technical creativity; types of technical creativity; activation methods; domestic methods; foreign methods; creative groups

\section{INTRODUCTION}

Today, technology solves the most important problems of mankind as a whole and is the basis of modern civilization. The role of the engineer and his creative activity is growing. But modern industrial production requires an everyday and effective process of searching for engineering ideas and solutions, technical creativity. Therefore, the question of the effectiveness of engineering activity and the forms of its organization is acute. An engineer, even a very talented one, can't constantly generate new technical ideas. Today there are no conditions for work of single-creators; there is no time to wait for the inventor to visit the inspiration. Neither can it large groups, as it takes some time to organize, agree, develop, etc. [1]. This problem is successfully solved on the basis of activation methods of technical creativity, which are both individual and collective. The development of activation methods of creative activity is being conducted all over the world, including Russia. In domestic and foreign practice, the activation methods are different, but despite this, their effectiveness in both cases is quite high. Therefore, a comparative analysis of domestic and foreign activation methods of technical creativity, their forms and methods of organization, conditions and features of their practical application is very relevant [2].

\section{CONCEPT OF THE TECHNICAL CREATIVITY}

Technical creativity is the process of creating new material means that increase the production efficiency. In technical creativity, the focus of attention is the solution of practical problems, when the rules and properties expressed through the laws of science are put into practice in technical devices. The result of technical creativity should correspond to such signs as novelty, practical significance, the possibility of tangible embodiment. These characteristics have a specific historical character, and hence a different ratio of creative and uncreative moments reflecting one or another level of technological development. These aspects should be taken into account when developing activation methods of technical creativity.

It should be noted that in practice, technical creativity and engineering creativity are often identified. And this is natural, since the main direction of the engineer activity is the development and improvement of technical systems, the search for new technical ideas and solutions meeting modern requirements not only of technical reliability but also environmental safety, and in this sense these concepts coincide. However, at the same time, modern engineering activity includes many other types of work: an executive engineer, an engineer-organizer, a design engineer, a production engineer, etc., that is, engineering creativity is wider than technical creativity. Technical creativity includes certain necessary stages and forms of work aimed at developing technical systems: research, improvement, innovation proposals, inventions and discoveries [3].

It is important to point out that the principles of technical creativity and the activation methods at different stages differ significantly. In this study, however, the authors have limited themselves to analyzing only some of the principles of technical creativity that can be used in research processes and in the improvement of technical systems.

\section{COMPARATIVE ANALYSIS OF DOMESTIC AND FOREIGN ACTIVATION METHODS OF TECHNICAL CREATIVITY}

Domestic activation methods of searching for engineering ideas and solutions, for technical creativity were 
developed by inventors on the basis of analysis of large practical material and are aimed at solving non-standard technical problems. In the domestic practice, it is believed that it is necessary to study the history, the logic of technical object structure, and the laws of technical systems development. Knowledge of regularities makes it possible to greatly narrow the field of search, to replace "guessing" with the scientific approach $[4,5]$. The development basis of the methods of searching for engineering ideas and solutions was a unique, patent fund available only in technology. The study and analysis of a large number (tens of thousands) of patents, author's certificates, and the study of the history and logic of the technical systems development made it possible to create a methodology for technical creativity, the theory of solving inventive problems, which was further improved and developed. In other words, based on the analysis of patent developments of varying degrees of complexity, typical techniques for improving the functional and constructive links of a technical object are revealed. Their application allowed improving the design as a whole. This list of methods of technical creativity is a kind of engineer-inventor reference [6]. It is clear that they cannot be represented as a specific set of "ready-made recipes". But they can act as a methodology, methods of searching for engineering ideas and solutions, activation methods of technical creativity.

Methods of searching for engineering ideas and solutions, activation methods of technical creativity, as a rule, are aimed at solving non-standard technical problems. At present, about 50 basic techniques for the activation methods of technical creativity have been developed, and together with various modifications they account for about 300 in number.

The appearance of activation methods is due to practical needs in the regular creativity process. Mastering the methods of searching for new technical solutions for a modern engineer is as important as using modern information technologies in his activities. The first work on this problem is connected with the name of the Bakuvian engineer G. S. Altshuller (1926-1998). As noted above, based on the generalization of a huge number of inventions, patent research, the laws of technical creativity were empirically determined, which allowed the creation of a theory of inventive problem solving (better known by its Russian acronym TRIZ) [7]. Along with the theory of inventive problem solving, an algorithm for inventive problem solving (ARIZ) appeared and developed, which at first was called "the technique of inventive creativity". This is a complex program of algorithmic type, designed for analysis and solution of inventive problems. The development of new ARIZ modifications was also based on the study of a large number of patent information on higher level inventions. The tasks of developing technical systems according to ARIZ are solved by identifying technical contradictions, determining the reason that caused these contradictions and eliminating them, i.e. resolving contradictions. Later ARIZ modifications have been significantly improved and represent an edifice of methods and techniques.

The theory of inventive problem solving (TRIZ) includes both theoretical and practical aspects. The theoretical foundations of TRIZ include the following issues: technology, its structure, technical creativity, technical systems and their structure, the development stages of technical systems, technical contradiction, increase of the ideality degree of technical systems, technical system deployment and reduction, increasing the agility and controllability of the technical systems and others.

Practical aspects of TRIZ include: typical methods of eliminating technical contradictions, substance-field analysis, and standards for solving inventive problems, solving atypical problems, using physical, chemical, and geometric and other effects and phenomena in solving inventive problems. Virtually this list of methods of technical creativity is widely used in engineering.

An important position of technical creativity activation in G.S. Altshuller technique is the technical contradiction. The fact is that in the process of technical creativity, when improving technical systems, a situation of technical contradiction constantly arises, which manifests itself in the form of deterioration of one or several properties of a technical system while improving certain characteristics of it. Such situation is observed in any technical system with its improvement. The unity of improvement and deterioration of various properties of the technical system in the improvement process is called a technical contradiction. Technical contradiction and resolution methods are very important for the direction of searching for new technical solutions, for developing activation methods of technical creativity. Technical contradiction is a necessary factor for identifying disadvantages that interfere with the functioning of an improved technical system.

Methods for resolving contradictions that are associated with certain stages of technical system modernization have also been developed. There are only five:

- The process of improvement is not connected with the contradiction elimination. The various parts of the mechanism and their coordination are being finalized;

- It includes small inventions obtained as a result of elimination of contradictions by traditional methods, standard for this industry, while changing, as a rule, only one element of the technical system;

- It is characterized by the overcoming of contradictions in ways known in the framework of one science (chemistry, physics, etc.). One of the system elements completely changes;

- A new technical system is created, in which the contradictions of the old are completely resolved, but it fulfills the former functions. Contradictions are eliminated by the of other sciences. For example, a physical problem is solved by chemical methods [8];

- A fundamentally new design is invented, which owes its appearance to discovery in science and technology. This can even lead to the appearance of a new industry. For example, the invention of radio contributed to the creation of radio engineering. 
In practice, as a rule, the process of development of technical systems is limited to the first 2-3 levels, it does not go on further [9]. To further improve or create a fundamentally new technology, other forms of technical creativity are required.

Domestic methods of technical creativity have their advantages and disadvantages. As mentioned above, the basis for the development of domestic methods is the analysis of the technology development laws and the processes of improving technical systems. The prevailing method is a logical approach. As it is known, logic and creativity are to some extent opposed and a rather complex analysis is required to reveal their unity. But this does not reduce the high efficiency of domestic methods in the development and improvement of technical systems.

Another approach that allows developing non-standard, new technical ideas is noted in foreign practice. In foreign activation methods of technical creativity all attention is usually focused on psychological aspects, on the psychology of invention, and hence on the activation of the psychological creativity moments (association, analogy, etc.). At the same time, great attention was paid to overcoming psychological inertia. In this case, by psychological inertia we mean the habit of template thinking, the desire to do "as always, like everyone else," and this is in most cases really necessary and justified. However, when searching for a new solution, psychological inertia is a serious obstacle, it hinders the non-standard approach, the new vision of the problem from different points of view [10]. The use of heuristic methods helps reduce the psychological barrier.

Activation methods in foreign practice are collective and individual. Creative groups are of particular interest among the collective forms in foreign practice. They can solve fairly complex tasks. Creative groups have found wide application in all industries abroad. A special advantage of creative groups is also the fact that participants with medium abilities can work productively here. Creative groups do not eliminate individual creativity, but, on the contrary, there is an increase in the intellectual capabilities of each participant. Here individual characteristics are united in a single process, as a result of which there is a special intellectual tension [11].

In this paper, we propose the forms of organization and work of creative groups developed by the French researcher Gi Aznar [12]. Our choice is due to the fact that this approach is the most effective and meaningful in practical terms. Limitations of work amount do not allow detailed analysis of work of creative groups. Our task is only to review the most common aspects of organization and work. It should be noted that the foreign experience of work of creative groups has not found its application in domestic practice. There are also no domestic developments in this direction.

In analyzing the organization and work of creative groups using Gi Aznar method, we will distinguish 8 elements, in our opinion, the most significant. These include: the principles of participants selection, the motivation and its importance, the number of participants, the schedule and the time of work, the factor of flexibility in searching for ideas and solutions, the psychological climate, the importance of the competitive moment in the group, the role of the leader.

Gi Aznar believes that the difference principle is an important factor in the selection of group members, the wider the circle of interests is and the more diverse the composition of the group is, the more productive it will work. A new idea, as a rule, arises at the intersection of knowledge from various fields. And creative procedures are connected with generation, processing, interaction of various information. In other words, each participant must be many-sided, possess a wide erudition and mental outlook. Further, for the group to be working, this diversity of the various characteristics of the participants must be brought to unity. Therefore, each participant undergoes a secondary selection, where the assessment is not only based on intellectual and personal qualities, but the question is whether the participant can successfully work in this group. In other words, the creative group should not represent a mechanical set of soloists, even if they are very talented, but a harmonious collective.

The second important characteristic of the group, according to Gi Aznar is motivation. At the very beginning when selecting a group, it is necessary to eliminate the possibility of false motivations. This means that the creative team should clearly know what is expected of it. Experience shows that the creativity process is of interest to all, but the motives may be different. The group is formed only in order to search for new solutions and be sure to achieve success. Participants with weak motivation at a certain stress, difficulties are disinhibited, and this significantly reduces the effect of group work.

The third important criterion for Gi Aznar is the number of participants in the group. A group with more than 10 partners tends to be divided into subgroups, and they are tempted to distract from solving problems, to be present, but not to work. In the course of numerous experiments, the greatest result is achieved by a group of 5-6 people. This strengthens the group cohesion, gives an opportunity to speak out to everyone, which is very important for productive work. Moreover, numerical limitations are associated with certain properties of human thinking, memory, perception, processing of simultaneous information, etc.

According to Gi Aznar, the fourth point of the creative group work concerns the time rhythms of the work. As the experimental data show, the most interesting ideas are expressed in two hours after the beginning of the work, then their number decreases, fatigue sets in, it seems that everything has been said, but after a while some creativity comes up, then there is a decline. As practice shows, the group can work productively only three hours a week. At the end of the session, there is a sense of exhaustion of solutions to this problem, but when the next session of creativity suggests to return to the previous topic, completely new ideas arise, and the old ones take a more thoughtful form, at the next session the same, that is, grows like a snowball. The group comes to the belief that there is a significant reserve of creativity, "wings grow", but this process should be managed by the leader. 
Of great importance for Gi Aznar also has such a characteristic of the group as flexibility. The creative group should be trained to "forget" the rigid formalized rules of the ordinary approach, clearly expressed logical structures, habitual reasoning. The inventive spirit is inseparable from audacity and often goes beyond the framework of this logic system. Creative thinking can't be limited to logical rigor, otherwise the researcher comes to a standstill. Wishing or not he seeks to exit from these logical forms, and therefore, moves on to new methods and then non-standard solutions appear. In Gi Aznar system, there is a whole series of methodical techniques for alternating opposite forms of activity - logic and creativity, which at the same time mutually complement each other. He emphasizes that these two approaches cannot be used simultaneously. In this case, they block each other, because they are opposite and assume completely different methodical techniques. Only awareness of the alternation of phases makes it possible to reveal the true meaning of each of them. One should not make a decision very quickly, since if the engineer has chosen any option, then the possibility of considering other solutions, even the best ones, is psychologically rejected.

Gi Aznar attaches importance to criterion, which characterizes the psychological climate of the creative group. The group should focus on a certain form of communication among them, paying attention to the following points: be yourself before others, do not pose, do not be afraid to make mistakes, seem ridiculous, express bold ideas, be considerate, listen to others, hear, react, and exclude speech phrases, utterances that destroy communication. This must be taught.

The seventh criterion of the creative group is the competitive moment. Gi Aznar is totally against the competitive moment, usually any comparisons slow down work, create an intolerant psychological climate.

Gi Aznar attached also great importance to the role of leader. The characteristics of the leader are related to the concepts of time and profitability. It means, during what period the group should give high results. Groups with an authoritarian leader are the fastest to achieve the best results. Groups with the opposite style of leadership (giving independence) have weak results, especially at the beginning, but after some time they catch up and get ahead of the "authoritarian" groups. There is a contradiction between the demands of early results and the desire to allow groups to reach the stage of self-organization. This contradiction can be smoothed over. To perform it, at first the leader must be authoritarian, and as the group organizes the work, they must shift to the opposite style of leadership.

Another approach, where the main factor in solving production problems is the differentiation principle of engineering activities, developed in the system of Colette Mathieu-Batsch [13]. This technique is the simplest, does not require much time for its implementation. Colette MathieuBatsch notes that for the functioning of the group it is necessary to take into account the specific features of the mental activity of individuals. Each researcher in his own way proceeds to solve this or that problem. Within this framework, there are specific and abstract approaches. None of them is more important than the other. Nevertheless, it is impossible in one group to confuse researchers with different object perceptions, as they will block each other's creative possibilities. But one can't confirm any advantage of this or that type of group, since each group individually has both advantages and disadvantages, which are absent in the opposite group. Therefore, in the process of solving the problem, it is necessary to use these two approaches in their unity. Both styles are necessary for a correct and effective problem solution.

The degree of application of activation methods of creative activity depends on the stage of solving problems, their complexity, that is, from the moment of its theoretical substantiation to its practical application [14].

The Colette Mathieu-Batsch system allows solving a wider range of production problems, including theoretical ones, in contrast to Gi Aznar method. The question, which one is better, is not discussed since their meaning and application is related to specific conditions and production tasks.

\section{CONCLUSION}

In this paper, only a few areas of development of activation methods of technical creativity in domestic and foreign practice are touched upon. Domestic and foreign methods have both their advantages and disadvantages. Domestic methods are more complex and require high qualification of engineering activities. Foreign methods, in particular creative groups, allow generating non-standard, new technical ideas to developers with average abilities, this is facilitated by the collective factor of creativity. In the domestic practice, unfortunately, the collective activation methods of technical creativity are not sufficiently developed. And as a recommendation, we should suggest using rational moments of both domestic and foreign methods, depending on the degree of complexity of the problem being solved, its specifics, and also take into account the peculiarities and creative capabilities of developers.

\section{REFERENCES}

[1] Inozemtsev V. A., Ivlev V. Y., Ivleva M.L. Artificial intelligence and problem of computer representation of knowledge. Proc. of the 2017 2nd International Conference on Contemporary Education, Social Sciences and Humanities. (ICCESSH 2017). Part of the series ASSEHR. Moscow, Russia, Vol. 124, p. 1151-1157.

[2] Ivlev Yu. V., Ivlev V. Y., Ivleva M.L. Logical-argumentative basics of educational culture. Proc. of 4th International Conference on Education, Language, Art and Intercultural Communication (ICELAIC 2017) Part of the series ASSEHR. Moscow, Russia, pp. 173-177.

[3] Potaptsev I.S., Bushueva V.V., Bushuev N.N. The main directions of technical creativity in engineering education. Proceedings of Higher Educational Institutions. Machine Building, 2014, no. 8, pp. 80-88.

[4] Gubanov N.I., Gubanov N.N. Subjective reality and space // Voprosy filosofii. 2015. No. 3. P. 45-54.

[5] Gubanov N.I., Gubanov N.N. The role of mentality in the development of society: sociocultural hypothesis // Vestnik slavianskikh kultur-bulletin of slavic cultures-scientific and informational journal. 2017. Vol. 43. No. 1. P. 38-51. 
[6] Altshuller G.S. Standard solutions of inventive tasks (76 standards). Available at: http://www.altshuller.ru/triz/standards.asp. Accessed April 19, 2018.

[7] Altshuller GS To find an idea: an introduction to TRIZ - the theory of inventive problem solving, Moscow, Albina Publisher, 2014.

[8] Lebedev S.A. The Structure of Scientific Rationality // Voprosy filosofii. 2017. No. 5. P. 66-79.

[9] Kravchenko I.I., Zavarzin V.I., Bushuev N.N., Smirnov S.G., Bushueva V.V. Analysis of the forms of technology practice for the preparation of qualified engineering personnel . Proceedings of Higher Educational Institutions. Machine Building, 2016, no. 11, pp. 40-49.

[10] Potaptsev I.S., Pavlikhin G.P., Bushuev N.N., Bushueva V.V. The use of foreign experience in solving tehnical problems in the engineering training of students: a handbook. Prof. V.A. Gorodnichev, ed. Moscow, International Publishing Center "Etnosotsium", 2015.

[11] Gubanov N.I., Gubanov N.N. Mentality: the nature and functioning in society // Voprosy filosofii. 2013. No. 2. P. 22-32.

[12] Aznar Gi. La creativite dans l'entrepise. Paris, 1971.

[13] Mathieu-Batsch Colette. Invitation a la creative. Paris, 1983.

[14] Oseledchik M.B., Ivlev V.Y., Ivleva M.L. Knowledge as a nonequilibrium dynamic system. Proc. of the 2017 2nd International Conference on Contemporary Education, Social Sciences and Humanities". (ICCESSH 2017). Part of the series ASSEHR. Moscow, Russia, Vol. 124, pp. 1-5. 